\title{
FoxO3a Modulates Hypoxia Stress Induced Oxidative Stress and Apoptosis in Cardiac Microvascular Endothelial Cells
}

\author{
Shenwei Zhang ${ }^{19}$, Yilin $\mathrm{Zhao}^{29}$, Ming $\mathrm{Xu}^{39}{ }^{3}$, Li Yu' ${ }^{1}$, Yujie Zhao ${ }^{1}$, Jianghong Chen ${ }^{4}$, Yiqiang Yuan ${ }^{1}$, \\ Qiangsun Zheng ${ }^{4}$, Xiaolin Niu ${ }^{4 *}$
}

1 Department of Cardiology, The Seventh People's Hospital of Zhengzhou, Zhengzhou, China, 2 Department of Respiration, Tangdu Hospital, The Fourth Military Medical University, Xi'an, China, 3 Department of Physiology, The Fourth Military Medical University, Xi'an, China, 4 Department of Cardiology, Tangdu Hospital, The Fourth Military Medical University, Xi'an, China

\begin{abstract}
Cardiac microvascular endothelial cells (CMECs) dysfunction induced by hypoxia is an important pathophysiological event in myocardium ischemic injury, whereas, the underlying mechanism is not fully clarified. FoxO transcription factors regulate target genes involved in apoptosis and cellular reactive oxygen species (ROS) production. Therefore, the present study was designed to elucidate the potential role of FoxOs on the hypoxia-induced ROS formation and apoptosis in CMECs. Exposure to low oxygen tension stimulated ROS accumulation and increased apoptosis in CMECs within 6-24 h. Hypoxia also significantly increased the expressions of HIF-1 $\alpha$ and FoxO3a. However, hypoxia decreased the phosphorylation of Akt and FoxO3a, correlated with increased nuclear accumulation. Conversely, the expression of FoxO1 was not significantly altered by hypoxia. After inhibition of HIF-1 $\alpha$ by siRNA, we observed that hypoxia-induced ROS accumulation and apoptosis of CMECs were decreased. Meanwhile, knockdown of HIF-1 $\alpha$ also inhibited hypoxia induced FoxO3a expression in CMECs, but did not affect FoxO1 expression. Furthermore, hypoxia-induced ROS formation and apoptosis in CMECs were correlated with the disturbance of $\mathrm{Bcl}-2$ family proteins, which were abolished by FoxO3a silencing with siRNA. In conclusion, our data provide evidence that FoxO3a leads to ROS accumulation in CMECs, and in parallel, induces the disturbance of Bcl-2 family proteins which results in apoptosis.
\end{abstract}

Citation: Zhang S, Zhao Y, Xu M, Yu L, Zhao Y, et al. (2013) FoxO3a Modulates Hypoxia Stress Induced Oxidative Stress and Apoptosis in Cardiac Microvascular Endothelial Cells. PLoS ONE 8(11): e80342. doi:10.1371/journal.pone.0080342

Editor: Masuko Ushio-Fukai, University of Illinois at Chicago, United States of America

Received June 26, 2013; Accepted October 2, 2013; Published November 20, 2013

Copyright: (C) 2013 Zhang et al. This is an open-access article distributed under the terms of the Creative Commons Attribution License, which permits unrestricted use, distribution, and reproduction in any medium, provided the original author and source are credited.

Funding: This work was supported by National Nature Science Foundation of China (No. 81200099) and Technological Innovation Grant from Tangdu Hospital, the Fourth Military Medical University (TDCX2011009). The funders had no role in study design, data collection and analysis, decision to publish, or preparation of the manuscript.

Competing Interests: The authors have declared that no competing interests exist.

*E-mail: faithword@163.com

9 These authors contributed equally to this work.

\section{Introduction}

Despite a wide range of therapeutic approaches, myocardial infarction (MI) continues to be a major cause of significant morbidity and mortality worldwide[1]. Coronary microcirculation dysfunction is a primary etiological component of myocardium ischemic injury. Cardiac microvascular endothelial cells (CMECs), comprising up to one-third of the total heart cells, play a critical role to keep coronary microvessels (diameter $<150 \mu \mathrm{m}$ ) and adjacent cardiomyocytes in normal condition[2]. Moreover, previous evidences suggest that CMEGs malfunction and apoptosis induced by hypoxia injury actually precedes cardiomyocyte apoptosis[3]. However, the mechanism of CMECs dysfunction induced by hypoxia injury is still not clear.

Oxidative stress, which is associated with increased formation of reactive oxygen species (ROS), contributes to the pathophysiology of hypoxia injury[4,5]. Increased ROS accumulation leads to proteins, nucleic acids and intracellular membranes modifications, subsequently inducing impairing cellular functions[6]. Furthermore, high level of ROS can also result in apoptosis by intrinsic death pathway[7]. Therefore,understanding the intracellular mechanism that regulates ROS formation is important to protect CMECs from hypoxic injury.

FoxO transcription factors, including FoxOl(FKHR), FoxO3 (FKHRL1), FoxO4 (AFX) and FoxO6, belong to the forkhead transcription factors, which have been implicated in regulating diverse cellular functions[8,9]. Previous studies have demonstrated that Akt, the serine/threonine kinase, phosphorylates FoxO transcription factors on distinct threonine and serine residues, which stimulate FoxOs export into the cytoplasm binding with 143-3 protein and eventual proteasomal degradation[10,11]. In addition to Akt, FoxOs can also be subjected to a complex regulation in response to starvation or oxidative stress. Previous study showed that FoxOl and FoxO3a, co-expressed in endothelial cells, are regulated by hypoxia [12]. Therefore, the present study was designed to dissect the mechanisms that regulate ROS formation and apoptosis and to elucidate the potential role of FoxOs on the hypoxic injury in CMECs. 


\section{Materials and Methods}

\section{Animals}

Adult male Sprague-Dawley (SD) rats (100-125 g) were purchased from the animal centre in the Fourth Military Medical University. Rats were housed in a temperature-controlled animal facility with a 12-hour light/dark cycle, with tap water and rodent chow provided ad libitum. The experiments were performed in adherence with the National Institutes of Health Guidelines on the Use of Laboratory Animals and were approved by the Fourth Military Medical University Committee on Animal Care.

\section{Isolation, Culture and Identification of CMEC}

CMECs were isolated according to a standard protocol as described previously[13]. Briefly, rats were euthanized by cervical dislocation after anesthesia with $5 \%$ isoflurane, and were placed in $70 \%$ ethanol for 5 minutes. Then, the hearts from adult male SD rats were removed under sterile conditions. After modifications, the left ventricle was immersed in $75 \%$ ethanol for $10 \mathrm{~s}$ to devitalize epicardial and endocardial endothelial cells. The tissue was minced and digested with $0.02 \%$ collagenase type II for $10 \mathrm{~min}$ and $0.025 \%$ trypsin for $10 \mathrm{~min}$ at $37^{\circ} \mathrm{C}$. After centrifugation at $1000 \mathrm{rpm}$ for $10 \mathrm{~min}$, the dissociated cells were resuspended in Dulbecco modified Eagle medium (Invitrogen, Cambridge, MA) supplemented with 15\% fetal calf serum (FCS) and plated on laminin $(10 \mathrm{mg} / \mathrm{mL})$ coated dishes.

GMECs were positively identified by Dil-acetylated low-density lipoprotein uptake assay. $3^{\text {rd }}$-passage CMECs were used for the further studies to avoid contamination with other cell types. After confluence, media were replaced to different conditions, including normal glucose medium $(5.5 \mathrm{mmol} / \mathrm{L})$ and high glucose medium (25 $\mathrm{mmol} / \mathrm{L})$.

\section{Hypoxia injury}

CMECs were stimulated with hypoxia injury as described previously[14]. Briefly, after being replaced by Hanks buffer, CMEGs were exposed to hypoxia $\left(94 \% \mathrm{~N}_{2}, 5 \% \mathrm{CO}_{2}, 1 \% \mathrm{O}_{2}\right)$ in an anaerobic system (Thermo Forma) at $37^{\circ} \mathrm{C}$ for $2 \mathrm{~h}, 4 \mathrm{~h}, 6 \mathrm{~h}$, $12 \mathrm{~h}$ and $24 \mathrm{~h}$ respectively. In the control group, CMEGs were maintained at normoxia (95\% air, $5 \% \mathrm{CO}_{2}$ ) condition.

\section{Measurement of ROS production in CMECs}

Generation of ROS in CMECs was visualized using the GENMED intracellular ROS red fluorescence determination kit (GENMED, Shanghai, China) according to the manufacturer's instructions. Then, 4,6-diamidino-2-phenylindole (DAPI) was stained for the identification of nucleus. Images were acquired and analyzed with Image-Pro Plus software version 6.0. The mean fluorescence intensity of each cell was calculated, and the total cell emission signals per field were averaged for data analysis.

\section{siRNA Transfection}

Cultured CMEGs were transfected with HIF- $1 \alpha$ siRNA (sense 5'-GGG AGU UGG AAGUAG UGG U), FoxO3a siRNA (sense 5'-GCU CUU GGU GGAUCA UCA A) or corresponding scrambled oligonucleotide control RNA for $6 \mathrm{~h}$ using Lipofectamine 2000 (Invitrogen) according to the manufacturer's instructions. Transduction efficiency was analyzed $24 \mathrm{~h}$ after transduction by flow cytometry and fluorescence microscopy.
Terminal deoxynucleotidy 1 transferase-mediated dUTP nick end-labeling (TUNEL) assay

The apoptosis of CMECs was confirmed by the TUNEL assay using an assay kit (In Situ Cell Death Detection Kit; Roche Diagnostics) according to the manufacturer's instructions[14]. Briefly, after hypoxia injury, CMEGs were incubated with TdT and fluorescein-labelled dUTP for $45 \mathrm{~min}$ at $37^{\circ} \mathrm{C}$ followed by DAPI staining for the identification of nucleus. The percentage of apoptotic cells was termed as apoptotic index. All of these assays were performed in a blinded manner.

\section{Immunofluorescence Staining for FoxO3a}

To determine FoxO3a localization in CMECs, immunofluorescence assay was performed as described previously[14]. Briefly, CMECs were fixed with $4 \%$ paraformaldehyde and permeabilized using $0.2 \%$ Triton $\mathrm{X}-100$ for $20 \mathrm{~min}$ at room temperature. CMECs were incubated with anti-FoxO3a antibody $(1: 200)$ at $4^{\circ} \mathrm{C}$ overnight followed by second antibody conjugated with FITC. Finally, DAPI was stained for the identification of nucleus. Images were acquired with a fluorescence microscope (NIKON Diaphot, Nikon, Tokyo, Japan).

\section{RNA extraction and Real-time Polymerase Chain Reaction (RT-PCR) analysis}

Total RNA was extracted from CMECs using TRIZOL reagent (Invitrogen) in accordance with the manufacturer's protocol. After reverse-transcribed, cDNA samples were synthesized using the Quantscript RT Kit (TianGen) and were subjected to polymerase chain reaction (PCR) amplification (see Methods $\mathrm{S} 1$ ).

\section{Western blot assay}

The expressions of HIF- $1 \alpha$, FoxO3a, FoxO1,BimEL and BclxL in CMECs were assessed by Western blotting following standard protocol[15]. Equal amounts of protein (50 $\mu \mathrm{g} /$ lane) were separated by electrophoresis on $12 \%$ SDS-PAGE gels for $90 \mathrm{~min}$ at $120 \mathrm{~V}$ and sequentially electrophoretically transferred to nitrocellulose (NC) membranes. After blocking, NC membranes were subjected to immunoblotting with primary antibodies over night at $4{ }^{\circ} \mathrm{C}$. After incubated with appropriate secondary antibody conjugated with horseradish peroxidase, blots bands were visualized with an enhanced chemiluminescene system (Amersham Bioscience). Densitometric analysis of Western blots was performed using VisionWorks LS, version 6.7.1.

The following primary antibodies were used: HIF-1 $\alpha$ (1:500, Santa Cruz Biotechnology), FoxO3a (1:1000, Cell Signaling Technology), FoxOl(1:1000, Cell Signaling Technology),BimEL(1:500, Cell Signaling Technology) and BclxL(1:500, Cell Signaling Technology). Secondary antibodies were horseradish peroxidase-conjugated goat anti-rabbit IgG (Santa Cruz Biotechnology) at 1:5000 dilution.

\section{Statistical analysis}

The results are presented as mean \pm SEM. Statistics were calculated using Prism 5.0 (GraphPad Software Inc, San Diego, CA, USA). Statistical comparisons for different groups were performed using either the Student's t test or one-way ANOVA. $p$ values $<0.05$ were considered statistically significant.

\section{Results}

Characterization of CMECs

Confluence CMEGs displayed typical "flagstone" morphology (Fig. 1A). Cells exhibited positive on Dil-acetylated low-density 

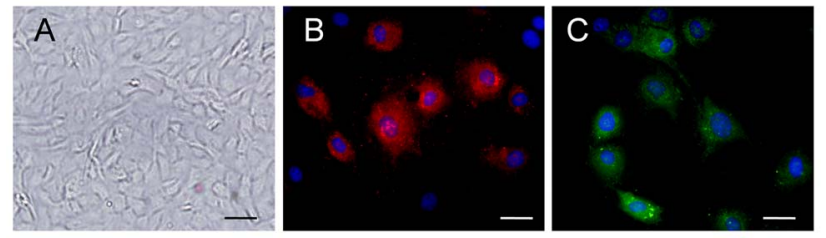

Figure 1. Characterization of CMECs. A, Confluent CMEC monolayer presents cobblestone morphology (scale bar:20 mm). B̧ CMECs uptook Dil-acetylated low-density lipoprotein (Dil-Ac-LDL) (red, Dil-AcLDL; blue, DAPI). C, CMECs express CD31. doi:10.1371/journal.pone.0080342.g001

lipoprotein intake assay (Fig. 1B). The representative immunofluorescence image demonstrated that CMEGs express CD31 (Fig. 1C).

\section{Hypoxia increased ROS production and apoptosis of CMECs}

ROS production, an index of oxidative stress, was measured to determine whether hypoxia induce oxidative stress in CMECs. The cells were exposed to hypoxic condition for $2 \mathrm{~h}, 4 \mathrm{~h}, 6 \mathrm{~h}, 12 \mathrm{~h}$ and $24 \mathrm{~h}$. Up-regulation of ROS was detectable $6 \mathrm{~h}$ after hypoxic condition and was consistently observed after exposure to hypoxia for 12 and $24 \mathrm{~h}$ (Fig. 2A).
Concurrently, TUNEL assay was performed to analyze the effect of hypoxia on the apoptosis of GMECs. As representative photomicrographs shown in Figure 2A, TUNEL-positive cells were more frequently observed after hypoxic incubation for $6 \mathrm{~h}$, $12 \mathrm{~h}$ and $24 \mathrm{~h}$. Quantitative analyses revealed that the apoptosis index in CMEGs was 9.33 $\pm 1.53 \%$ under normoxic condition. The apoptosis index in CMECs under hypoxia for $2 \mathrm{~h}$ and $4 \mathrm{~h}$ was $11.67 \pm 1.52 \%$ and $12.73 \pm 1.43 \%$ respectively, which was higher than that in normal group but without significance $(p>0.05)$. However, the apoptosis index in CMECs under hypoxia for $6 \mathrm{~h}, 12 \mathrm{~h}$ and $24 \mathrm{~h}$ was $28.07 \pm 3.61 \%, 33.13 \pm 2.03 \%$ and $36.43 \pm 1.51 \%$ respectively, significantly higher than that in normxic group $(p<0.05)$. These data suggested that hypoxia enhanced ROS accumulation in CMECs with increased apoptosis.

\section{Hypoxia regulated the expression and intracellular} localization of FoxO3a

To investigate whether hypoxia affect the steady expressions of FoxOs and HIF- $1 \alpha$, we analyzed the levels of FoxO 1, FoxO3a and HIF- $1 \alpha$ in CMEGs under hypoxia and normoxia condition. Western blot assay revealed that hypoxia led to a significant increase in the expressions of FoxO3a and HIF-1 $\alpha$ within 6-24 h, whereas the FoxOl expression remained essentially unaltered (Fig. 3A,B,C). FoxO transcription factors can be phosphorylated by Akt which stimulate FoxO3a exporting from the nucleus, binding with 14-3-3 protein, and eventual proteasomal degradation. Therefore, we evaluated the phosphorylated status of Akt and
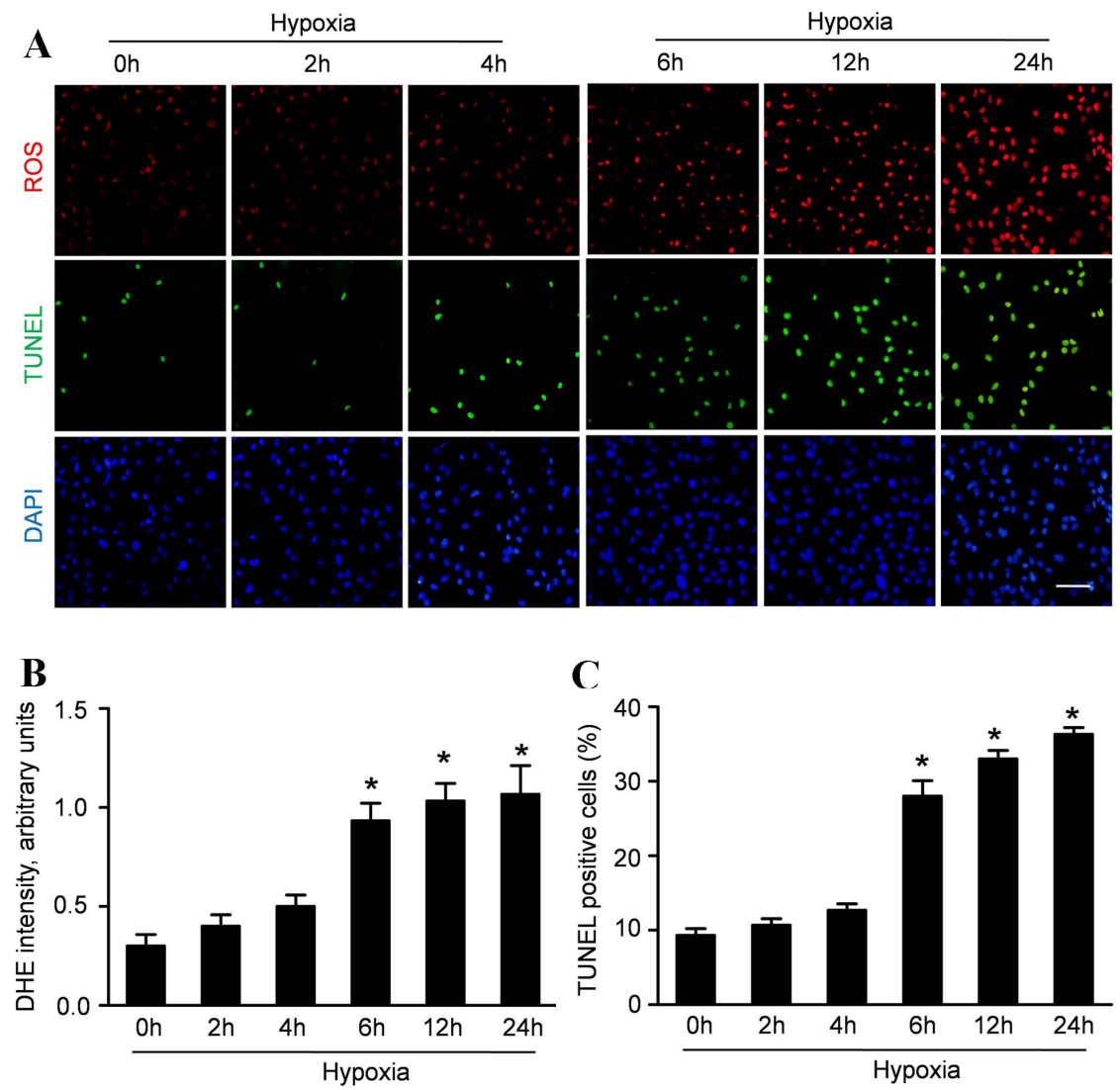

Figure 2.Hypoxia increases ROS production and apoptosis of CMECs. A: Representative immunofluorescence imagines of ROS formation (red fluorescent), apoptosis (TUNEL, green fluorescent) and DAPI (blue fluorescence) in CMECs under hypoxia condition for the indicated time points. (Scale bars: $20 \mu \mathrm{m}$ ) B: The average fluorescence intensity of DHE in each group. C: Quantification of the apoptotic CMECs was presented as the percentage of apoptotic cells. $\left(n=5,{ }^{*} p<0.05\right.$ vs. control). doi:10.1371/journal.pone.0080342.g002 

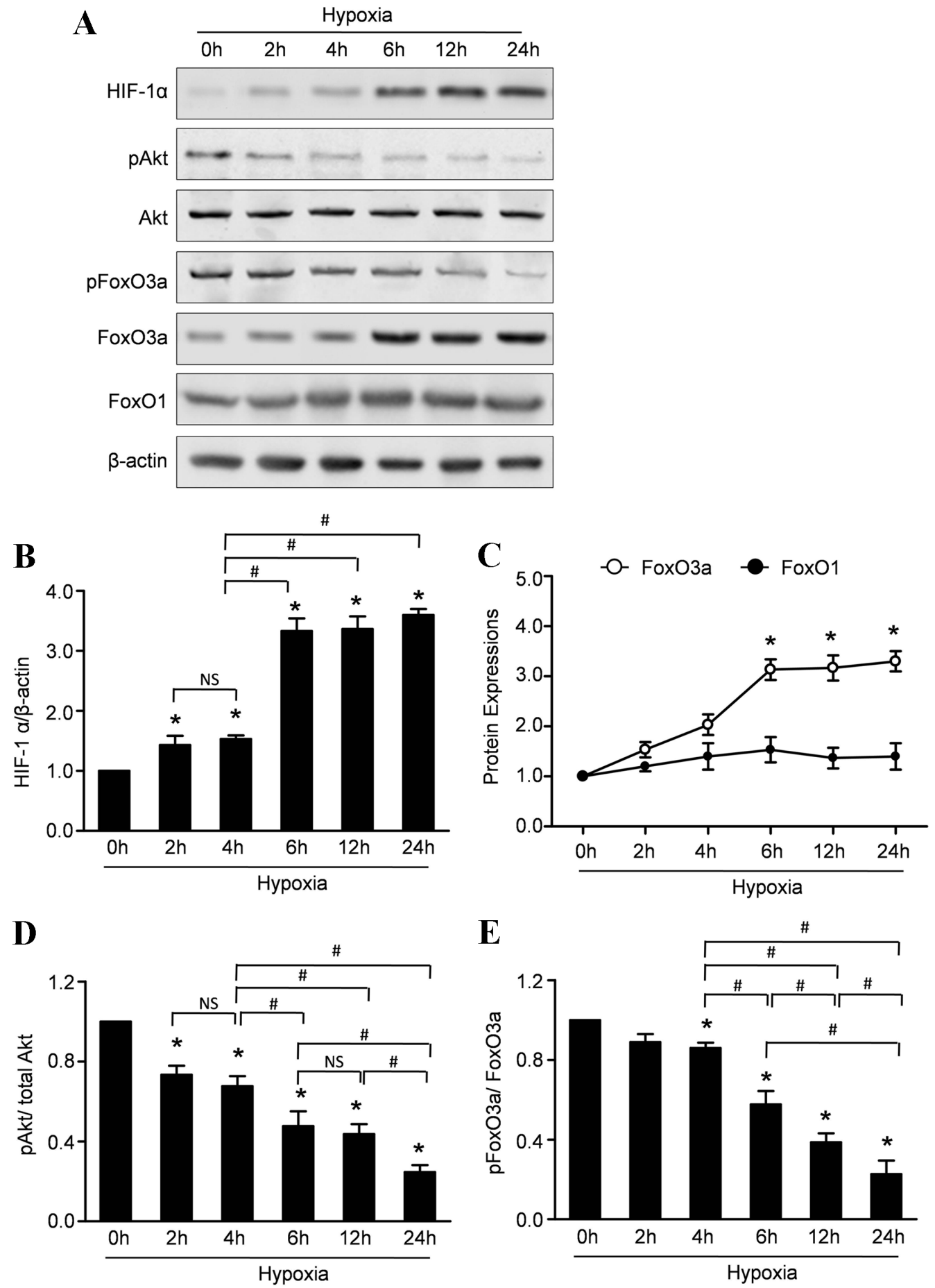

Figure 3. Hypoxia increases the expressions of HIF-1 $\alpha$, decreased the activity of Akt and regulates the expressions of FoxOs in CMECs. A: Representative Western blots of HIF-1 $\alpha$, pAkt, Akt, FoxO1, FoxO3a, pFoxO3a in CMECs subjected to hypoxic injury. B: Semiquantitative analysis of HIF-1 $\alpha$. C: Semiquantitative analysis of FoxO1 and FoxO3a at the indicated time point. D: The ratios of p-Akt/total Akt at the indicated time point. E: The ratios of $p$-Akt/total Akt at the indicated time point. $\left(n=5,{ }^{*} p<0.05\right.$ vs. control, \# $p<0.05$, NS: not significant). doi:10.1371/journal.pone.0080342.g003

FoxO3a to investigate whether hypoxia affects their activations. Representative results and quantitative analyses (Figure 3A, D and E) revealed that the expression of phospho-Akt was significantly decreased in the CMECs under hypoxic condition. Moreover, hypoxia also decreased the expression of p-FoxO3a, indicating that hypoxia impaired Akt activation followed with decreased phosphorylations of FoxO3a. Furthermore, immunofluorescence stain was performed to analyze the subcellular localization of FoxO3a. In normoxia condition, the FoxO3 protein was mainly localized in the cytoplasm with only $5.9 \%$ of the protein detectable in the nuclear fraction. Consistent with earlier studies in neuronal cells, hypoxia caused rapid nuclear accumulation of FoxO3a in 
A
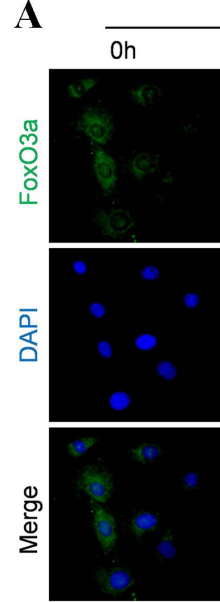

Hypoxia
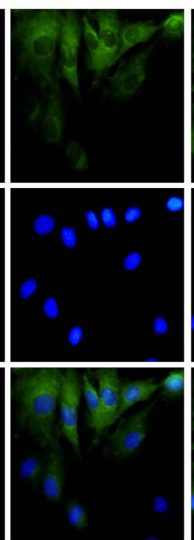

$4 \mathrm{~h}$
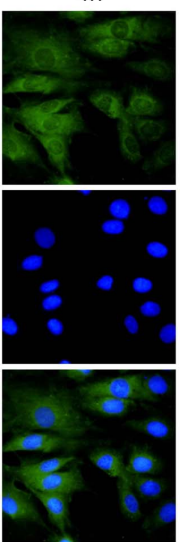

Hypoxia

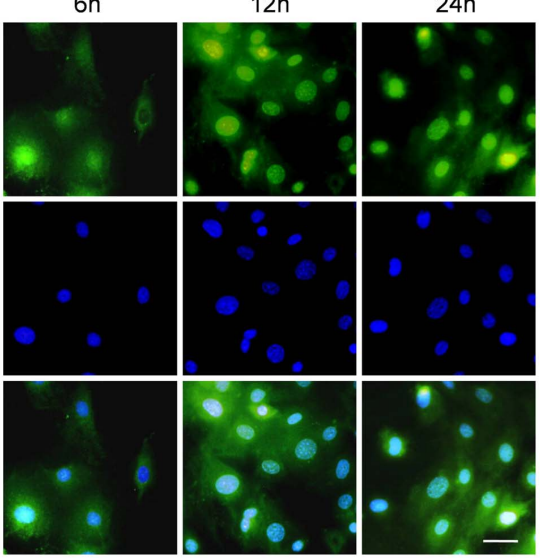

\section{B}

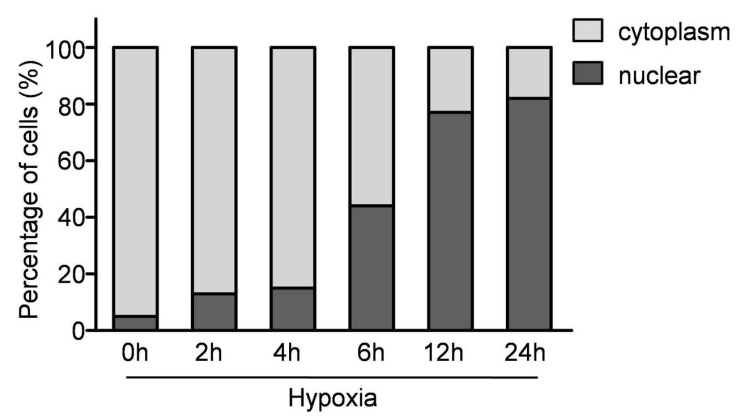

Figure 4. Hypoxia promoted FoxO3a nuclear location. A: Representative immunofluorescence staining for subcellular localization of FoxO3a (green fluorescent) in CMECs under hypoxia condition for the indicated time points. (Scale bars: $20 \mu \mathrm{m}$ ) B: Quantification of FoxO3 nuclear or cytoplasmic localization in CMECs $(n=5)$.

doi:10.1371/journal.pone.0080342.g004

CMEGs within 6 to $24 \mathrm{~h}$ (Fig. 4A). Quantitative analyses revealed that the percentage of CMECs with nuclear localization of FoxO3a was $44 \%, 77 \%$ and $82 \%$ after hypoxia injury for $6 \mathrm{~h}$, $12 \mathrm{~h}$ and $24 \mathrm{~h}$ respectively (Fig. $4 \mathrm{~B}$ ).

\section{HIF-1 $\alpha$ modulated the hypoxia-induced FoxO3a expression in CMECs}

To assess the contribution of HIF- $1 \alpha$ to hypoxia-mediated FoxO transcription factors expression, we used siRNA to inhibit the activation of HIF- $1 \alpha$. Western blot results revealed that HIF$\alpha$ rapidly accumulated in CMEGs under hypoxia condition which was reduced by more than $90 \%$ after transiently transfected with HIF- $1 \alpha$ siRNA (Fig. 5A,B). Real-time PCR results demonstrated that hypoxia increased the mRNA level of FoxO3a which was abolished by HIF- $1 \alpha$ silencing, indicating that HIF- $1 \alpha$ increased the transcription of FoxO3a. Moreover, the hypoxia-increased mRNA levels of Bim and Bcl-xl were also abolished by HIF- $1 \alpha$ silencing (Figure-S1). Concomitantly, inhibition of HIF- $1 \alpha$ by siRNA abolished the increased total FoxO3a protein expression (Fig. 5A,C) in hypoxia-mediated CMECs without affecting the protein level of FoxOl (Fig. 5A,F). These data indicated that the hypoxia induced FoxO3a expression in CMECs was modulated at least in part by HIF$1 \alpha$. Moreover, HIF- $1 \alpha$ silencing also abolished the hypoxiainduced increase of Bim expression and decrease of Bcl-xl level (Fig. 5A,G,H). Furthermore, we evaluated the effects of HIF-1 $\alpha$ on the phosphorylated FoxO3a. The representative Western results and quantitative analyses revealed that hypoxia significantly decreased the ratios of phospho-Akt/total Akt and phospho-FoxO3a/total FoxO3a, which were not affected by HIF-1 $\alpha$ (Fig. 5A,D,E). These data indicated that HIF-1 $\alpha$ increased the transcription of FoxO3a which results in hypoxia increased FOXO3a protein synthesis.

\section{HIF-1 $\alpha$ contributed to the hypoxia induced ROS} accumulation and apoptosis of CMECs

To get an insight into the role of HIF- $1 \alpha$ on the hypoxic injury of CMEGs, we assessed the ROS accumulation and apoptosis of CMECs transfected with HIF- $1 \alpha$ siRNA. Inhibition of HIF- $1 \alpha$ did not alter ROS production under normoxia, whereas significantly reduced ROS accumulation by $50 \%$ in CMEGs under hypoxia (Fig. 6A, G). Meanwhile, inhibition of HIF-1 $\alpha$ decreased the apoptosis of CMECs under hypoxia, without affecting the apoptosis of CMEGs under normal condition (Fig. 6B). Quantitative analyses revealed that HIF- $1 \alpha$ siRNA did not alter the apoptosis index in CMECs under normoxia (10.01 $\pm 1.02 \%)$, compared with control siRNA $(8.03 \pm 1.12 \%, \mathrm{p}>0.05)$. However, the apoptosis index of hypoxic CMECs with HIF- $1 \alpha$ siRNA $(15.33 \pm 1.53 \%)$ was significantly decreased compared with control siRNA $(28.33 \pm 3.21 \%, \mathrm{p}<0.05)$ (Fig. 6D). These data indicated that HIF- $1 \alpha$ contributed to the hypoxia induced ROS accumulation and apoptosis of CMECs. 

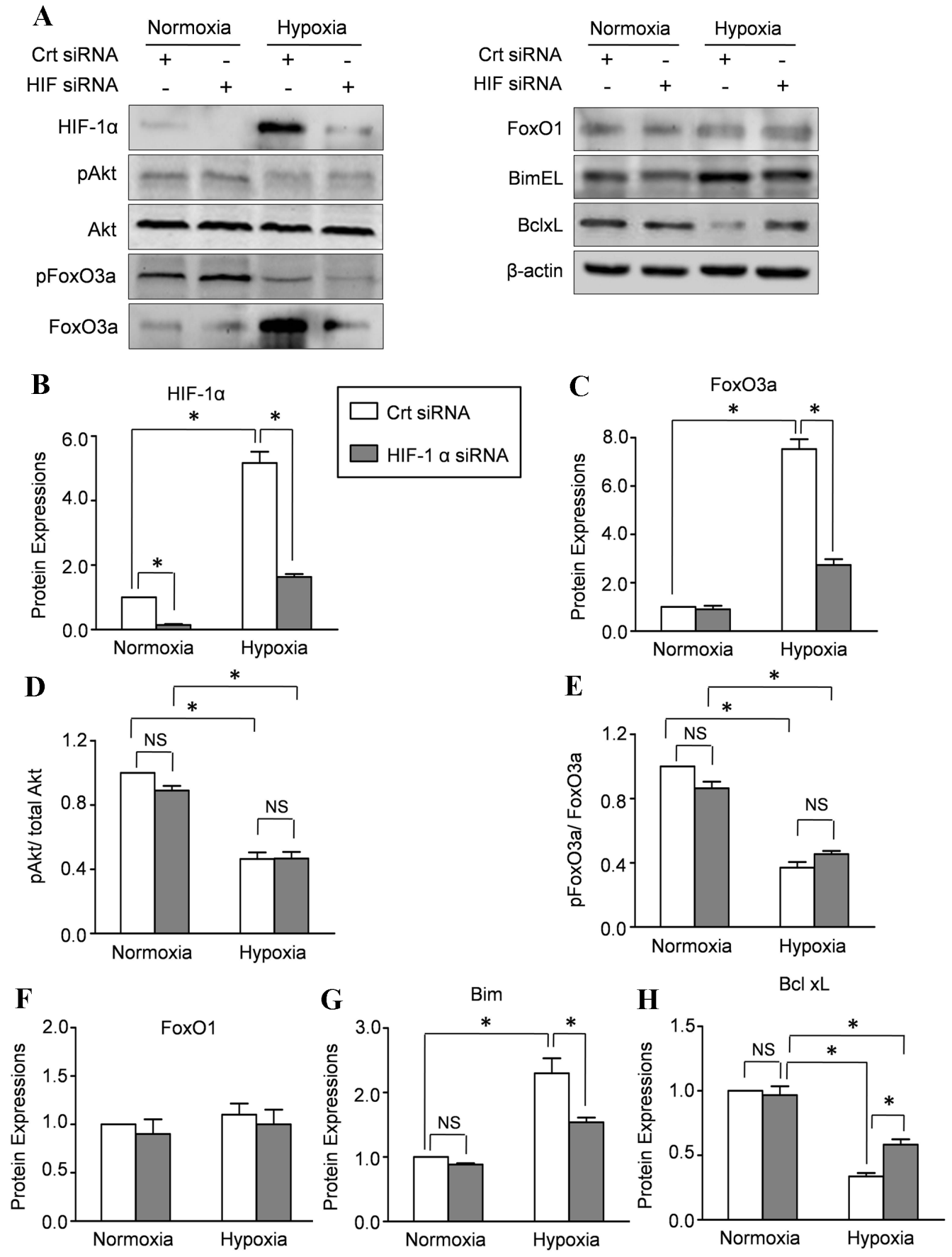

Figure 5. HIF-1 $\alpha$ modulated the hypoxia-induced FoxO3a expression in CMECs. A: CMECs were incubated with siRNA directed against HIF$1 \alpha$ (HIF-1 $\alpha$ siRNA) or scrambled oligonucleotide control RNA (Crt siRNA) and then subjected to hypoxia for $6 \mathrm{~h}$. Western blot assay was performed to evaluate the expressions of HIF-1 $\alpha$, p-Akt, Akt, FoxO1, p-FoxO3a, FoxO3a, BimEl and Bclxl. The semiquantitative analysis of expressions of HIF-1 $\alpha$ (B), FoxO3a (C), FoxO1 (F), BimEl (G) and BclxI (H). D: the ratio of p-Akt/total Akt. E: the ratio of p-FoxO3a/total FoxO3a. The protein expression in CMECs treated with control siRNA (Crt siRNA) under normoxia condition was set as $100 \% .\left(n=5,{ }^{*} p<0.05\right.$, NS: not significant). doi:10.1371/journal.pone.0080342.g005

\section{Endogenous FoxO3a affects the expressions of $\mathrm{Bcl}-2$ family in hypoxic CMECs}

The Bcl-2 proteins family regulates the process of apoptosis by the balance of pro- and anti-apoptotic proteins in mitochondria. Therefore, we performed Real-time PCR to evaluate the potential effect of FoxO3a on the mRNA level of Bcl-2 family. Our results revealed that FoxO3a silencing increased the mRNA level of Bcl-2 and Bcl-xl in CMECs under normal or hypoxia conditions. By contrast, FoxO3a silencing decreased the mRNA expression of Bim and Bax, indicating that FoxO3a regulated the transcription levels of Bcl-2 protein family (Figure-S2). Meanwhile, Western blot results revealed that FoxO3a siRNA transfection significantly decreased FoxO3a expression in GMECs under hypoxia and normoxia condition, whereas FoxOland HIF- $1 \alpha$ expressions remained essentially unaltered (Fig. 7A-C). Con- 

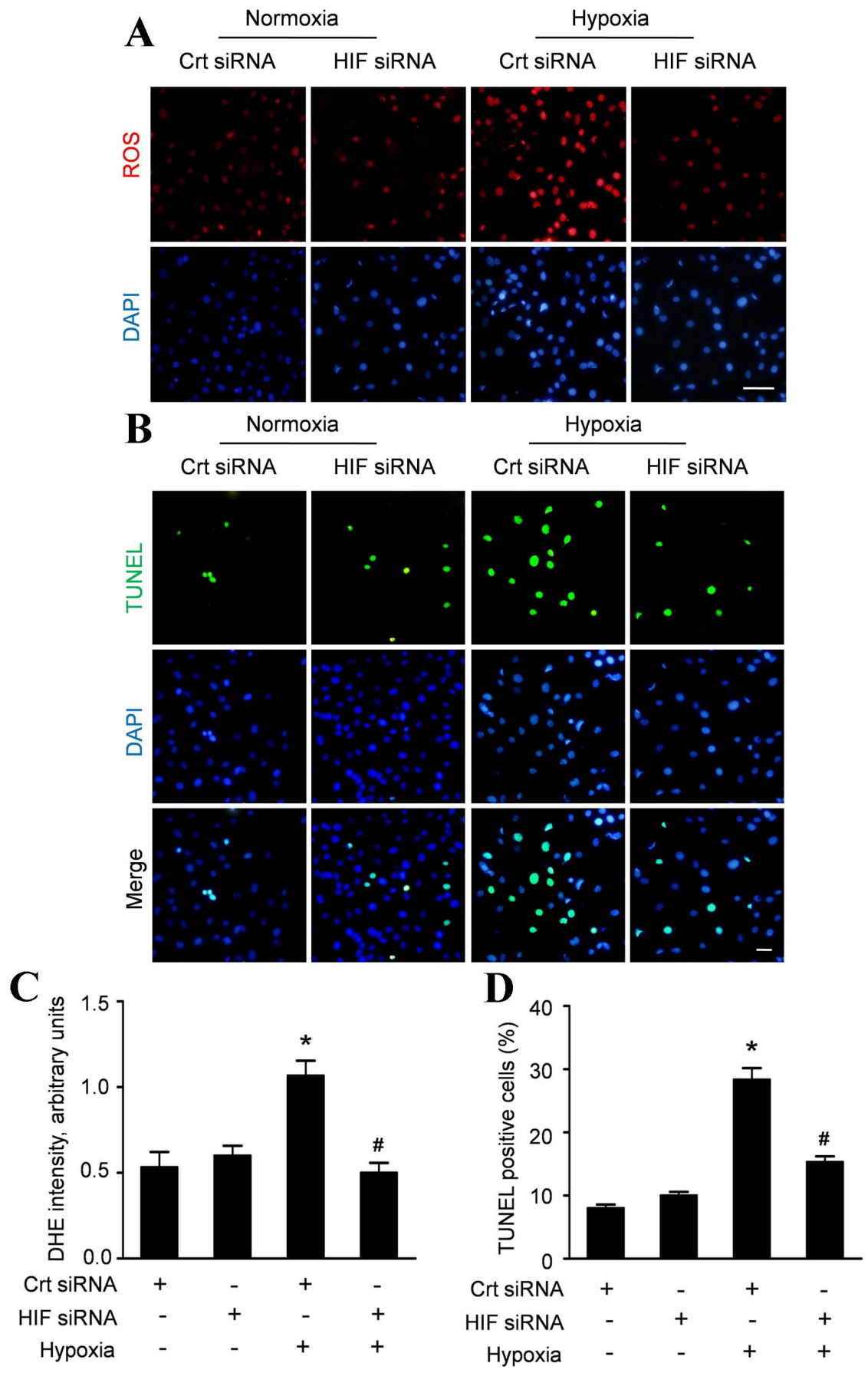

Figure 6. HIF-1 $\alpha$ regulates the hypoxia-induced ROS accumulation and apoptosis in CMECs. A: Representative immunofluorescence imagines of ROS formation (red fluorescent) and DAPI (blue fluorescence) in CMECs incubated with HIF-1 $\alpha$ siRNA or control siRNA (Crt siRNA) under normoxia or hypoxia condition for $6 \mathrm{~h}$. (Scale bars: $20 \mu \mathrm{m}$ ); B: Confocal imaging of cell apoptosis determined by TUNEL assay. Apoptotic nuclei were identified as TUNEL positive (green fluorescent) and total nuclei by DAPI counters taining (blue fluorescent). Scale bar represents $50 \mu \mathrm{m}$. C: The average fluorescence intensity of DHE in each group. D: Quantification of the apoptotic CMECs was presented as the percentage of apoptotic cells. $\left(\mathrm{n}=5,{ }^{*} p<0.05\right.$ vs. Crt siRNA plus normoxia, $\# p<0.05$ vs. Crt siRNA plus hypoxia).

doi:10.1371/journal.pone.0080342.g006

comitantly, hypoxia increased pro-apoptosis protein Bim EL and Bax with decreased expressions of anti-apoptosis proteins BclxL and Bcl-2. Silencing of FoxO3a by siRNA increased the expression of Bcl-2 and BclxL (Fig. 7A, E-H). However, hypoxia -dependent up-regulation of Bim EL and Bax was mainly affected by depletion of FoxO3a. These data suggested that FoxO3a is responsible for the hypoxia induced disturbance of Bcl-2 family proteins. 

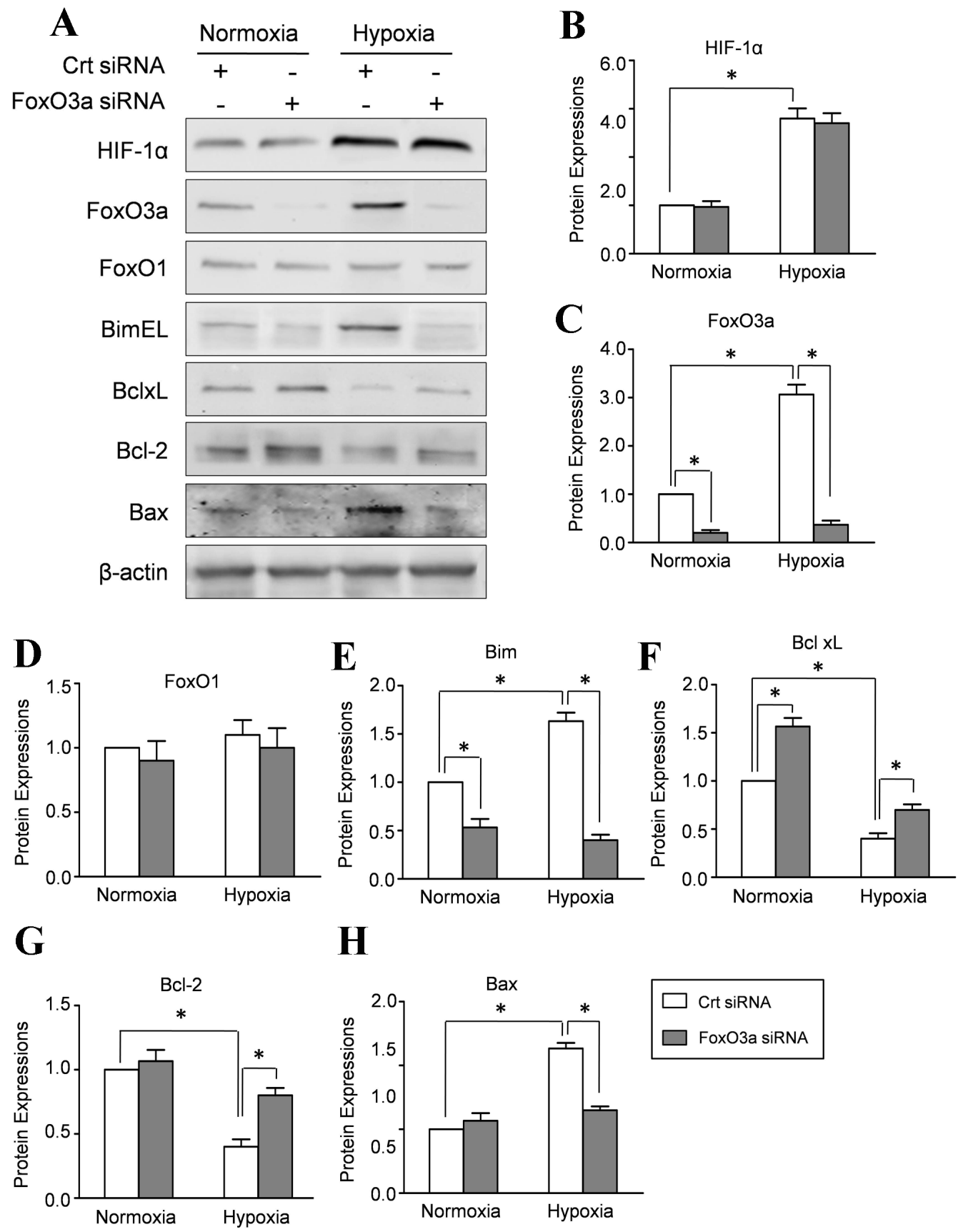

Crt siRNA

FoxO3a siRNA

Figure 7. FOXO3a affects the expressions of Bcl-2 family proteins in CMECs. CMECs were incubated with siRNA directed against FoxO3a (FoxO3a siRNA) or scrambled oligonucleotide control RNA (Crt siRNA) and then subjected to hypoxia for $6 \mathrm{~h}$. A: Representative Western blot of HIF$1 \alpha$, FoxO1, FoxO3a and $\mathrm{BCl}-2$ proteins, including Bim EL, Bcl xl, Bcl-2 and Bax. The semiquantitative analysis of HIF-1 $\alpha$ (B), FoxO1 (C), FoxO3a (D), Bim $\mathrm{EL}(\mathrm{E}), \mathrm{BCl} \times \mathrm{xl}(\mathrm{F}), \mathrm{BCl}-2(\mathrm{G})$ and Bax $(\mathrm{H})$. The protein expression in CMECs treated with control siRNA (Crt siRNA) under normoxia condition was set as $100 \%$. $\left(\mathrm{n}=5,{ }^{*} p<0.05\right)$.

doi:10.1371/journal.pone.0080342.g007

\section{FoxO3 silencing causes a decline in $\mathrm{ROS}$ production and} apoptosis induced by hypoxia

In line with the hypothesis that FoxO3a is crucially involved in the ROS production in hypoxic CMECs, we analyzed the ROS accumulation and apoptosis of CMECs transfected with FoxO3a siRNA. As shown in figure $8 \mathrm{~A}$ and $\mathrm{C}$, FoxO3a silencing did not alter ROS production under normoxia, whereas significantly reduced ROS accumulation in CMEGs under hypoxia. Similar results were observed in hypoxia induced apoptosis. Inhibition of
FoxO3a had an attenuating effect on the apoptosis of CMECs under hypoxia (Fig. 8B). Quantitative analyses in figure 8D revealed that FoxO3a siRNA did not alter the apoptosis index in CMECs under normoxia $(12.12 \pm 1.01 \%$ vs.10.67 $\pm 1.53 \%$, $p>0.05)$. However, the apoptosis index of hypoxic CMECs transfected with FoxO3a siRNA was significantly decreased compared with control siRNA $(18.02 \pm 1.21 \%$ vs.35.37 $\pm 1.52 \%$, $p<0.05)$. These data indicated that FoxO3as may be responsible for cellular ROS fluctuations and apoptosis induced by hypoxia. 

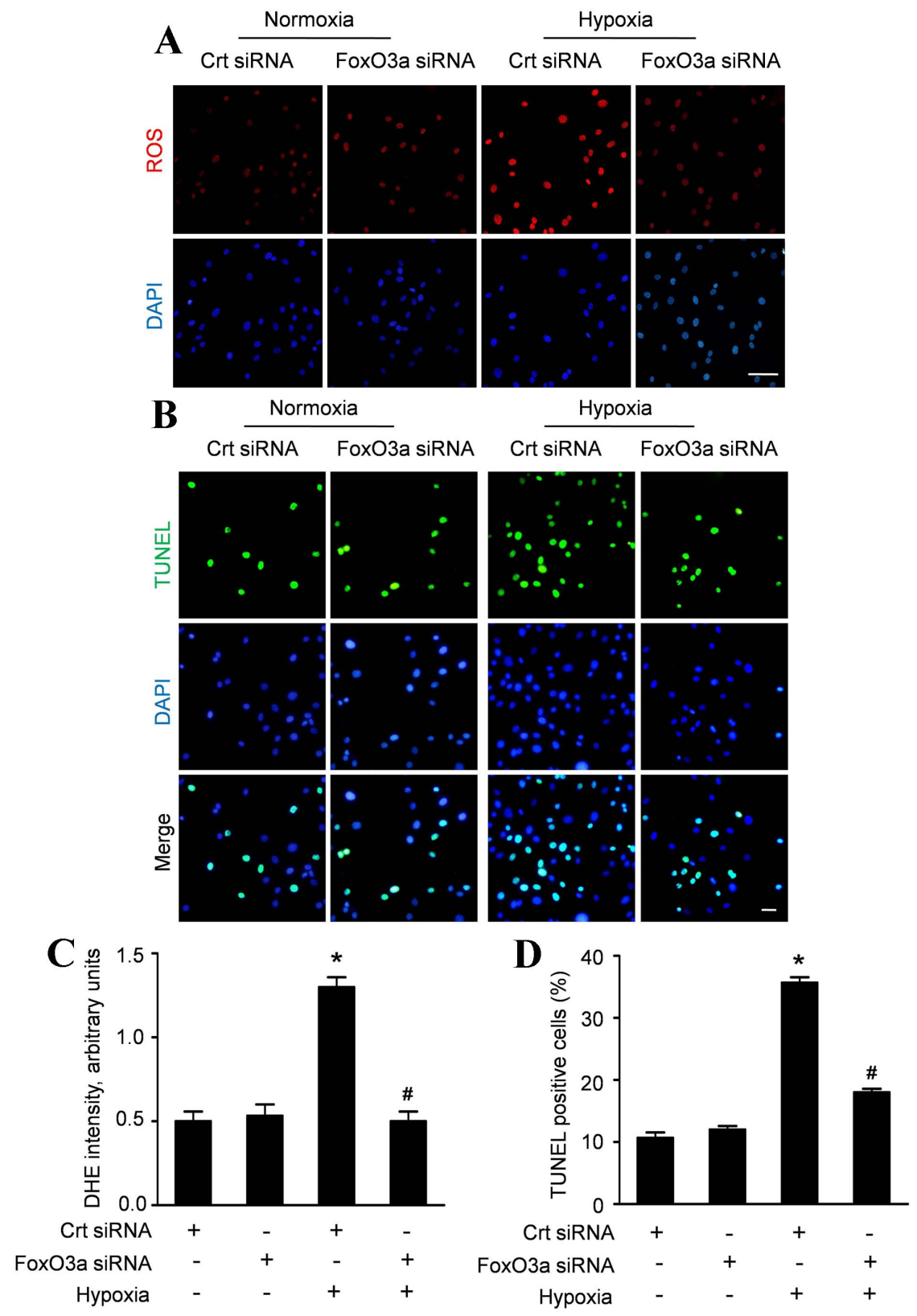

Figure 8. FoxO3 silencing suppresses hypoxia-induced ROS production and apoptosis in CMECs. A: Representative immunofluorescence imagines of ROS formation (red fluorescent) and DAPI (blue fluorescence) in CMECs incubated with FoxO3a siRNA or control siRNA (Crt siRNA) under normoxia or hypoxia condition for $6 \mathrm{~h}$. (Scale bars: $20 \mu \mathrm{m}$ ); B: Representative confocal imaging of TUNEL (green fluorescent) and DAPI (blue fluorescent) staining assay. (Scale bars: $20 \mu \mathrm{m}$ ) C: The average fluorescence intensity of DHE in each group. D: Quantification of the apoptotic CMECs was presented as the percentage of apoptotic cells. ( $n=5,{ }^{*} p<0.05$ vs. Crt siRNA plus normoxia, $\# p<0.05$ vs. Crt siRNA plus hypoxia). doi:10.1371/journal.pone.0080342.g008

\section{Discussion}

It is well recognized that CMECs apoptosis induced by hypoxia injury is an important pathophysiological event in myocardium ischemic injury[2]. However, the underlying mechanism of CMECs hypoxic injury is still not fully understood. In the present study, we demonstrate for the first time that HIF- $1 \alpha$ and FoxO3a play the central role in hypoxic injury of CMECs. Our data revealed that HIF- $1 \alpha$ increases the expression of FoxO3a, but not FoxOl under hypoxic stress. Furthermore, the activation of the transcription factor FoxO3a leads to ROS accumulation, and in parallel, induces the disturbance of Bcl-2 family proteins which are critical mediators of apoptosis in CMECs (Fig. 9).

The underlying mechanism of hypoxic injury is not fully understood. HIF- $1 \alpha$ leads to the activations of multiple proteins which regulate various physiological functions under hypoxia stress[16]. Furthermore, ROS have been demonstrated to mediate the proteolytic degradation of HIF- $1 \alpha$, indicating that HIF-l $\alpha$ level is regulated by the intracellular redox state[17]. Moreover, inhibition of ROS formation decreased HIF- $1 \alpha$ protein expression[18]. These results demonstrated that ROS directly link HIF$1 \alpha$ expression. Consistently, we found that hypoxia increased ROS formation and the expression of HIF-l $\alpha$ in the present study. 


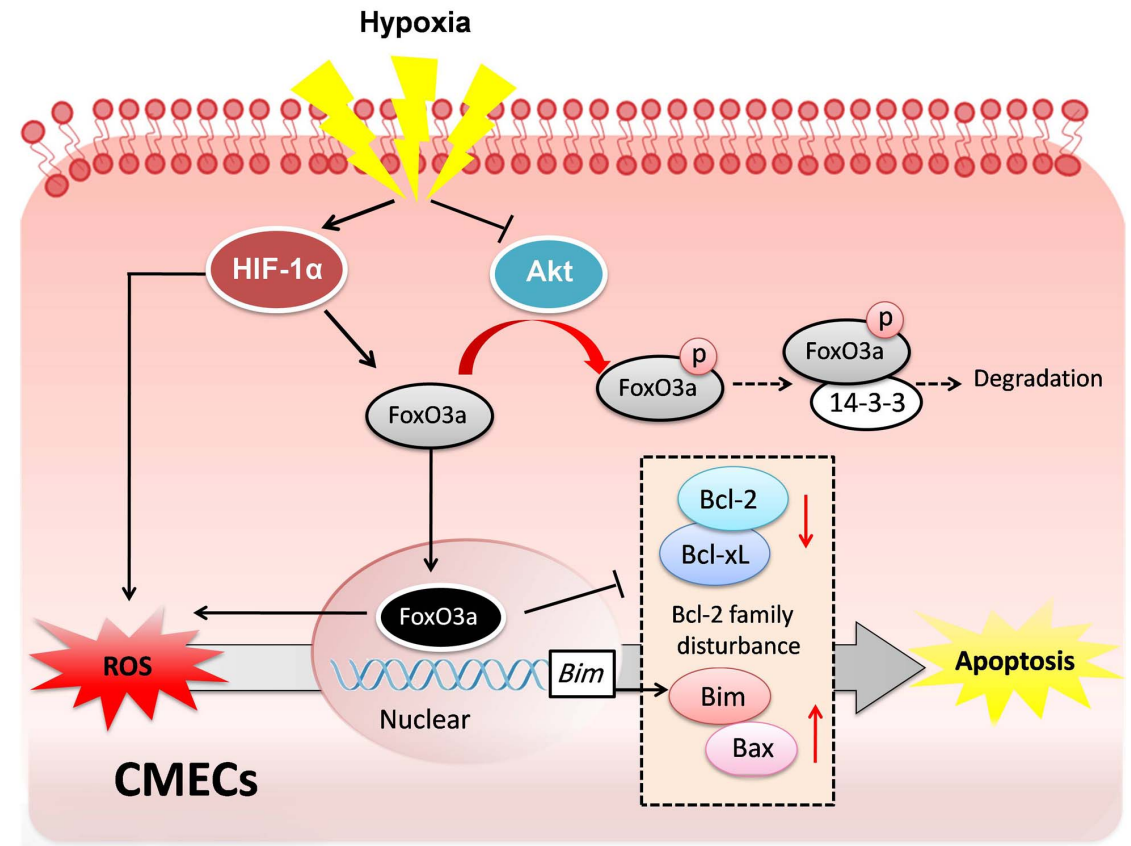

Figure 9. Proposed scheme for the mechanism that regulates hypoxia-induced injury in CMECs. Hypoxia stress increases HIF- $1 \alpha$ expression, which subsequently promotes the transcription of FoxO3a and directly stimulates ROS formation. Meanwhile, hypoxia also inhibits the activation of Akt which results in decreased phosphorylation and degradation of FoxO3a. Thus, hypoxia increases FoxO3a translocation to nucleus and the transcription activities, which results in the elevated ROS accumulation and Bcl-2 protein family disturbance. In parallel, increased ROS formation also promotes the activity of FoxO3a which increases the pro-apoptotic proteins Bim and Bax and decreased the anti-apoptotic proteins $\mathrm{BCl}-\mathrm{xL}$ and $\mathrm{BCl}-2$. The disturbance of anti-apoptosis proteins and pro-apoptosis proteins induced by oxidative stress subsequently triggers the apoptosis of CMECs. Accordingly, FoxO3a plays a central role in hypoxia-induced ROS formation and apoptosis in CMECs.

doi:10.1371/journal.pone.0080342.g009

Meanwhile, HIF- $1 \alpha$ silencing abolished the hyoxia-induced ROS formation, associated with decreased apoptosis in CMECs. Based on these results and previous published studies, we speculated of HIF- $1 \alpha$ in the hypoxic injury to CMECs.

FoxO factors are key regulators of target genes involved in cell cycle, apoptosis and differentiation $[8,19]$. Moreover, FoxO factors also regulate the cellular response to oxidative stress[20]. In this study, we assessed the expressions of both FoxO3a and FoxOl in CMECs and identified a novel connection between FoxOs and hypoxia injury. Our results revealed that hypoxia resulted in a significant increase of the expressions of FoxO3a. Interestingly, the FoxOl expression remained essentially unaltered in CMECs under hypoxia, indicating that FoxOl might not be required in the hypoxic injury. FoxO transcription factors can be phosphorylated by Akt. These phosphorylations stimulate FoxO3a exporting from the nucleus, binding with 14-3-3 protein, and eventual proteasomal degradation. In the previous study, our results revealed that hypoxia significantly decreased the expressions of phospho-Akt and phospho-FoxO3a, indicating that hypoxia impaired Akt activation followed with decreased phosphorylations of FoxO3a. Moreover, hypoxia also affected the intracellular localization of FoxO3a. FoxO3a were localized predominantly in the cytoplasm in CMECs under normoxia, indicating that FoxO3a was inactive with proteasomal degradation. However, hypoxia increased FoxO3a nuclear location, associated with HIF$1 \alpha$ activation, which is consistent with previous studies[12].

Although the mechanism by which hypoxia increases FOXO3a synthesis is still unclear. A direct protein-protein interaction of HIF- $1 \alpha$ and FoxO3a has been demonstrated in previous study, suggesting that FoxO3a may be a direct target of HIF-1 $\alpha$. Consistently, our results revealed that hypoxia increased the
mRNA level and total protein expression of FoxO3a, which were both abolished by HIF- $1 \alpha$ silencing. Conversely, HIF- $1 \alpha$ silencing did not affect the FoxOl expression. Moreover, there was no difference of the phosphorylated FoxO3a expression in CMECs with or without HIF- $1 \alpha$ silencing, indicating that HIF- $1 \alpha$ had no effect on the phosphorylation statuse of FoxO3a. Therefore, these data indicate that HIF- $1 \alpha$ increases the FOXO3a protein synthesis by promoting the transcription activity.

Physiological levels of intracellular ROS generation act as second messengers, which are deemed necessary for normal cellular function. Excessive ROS generation may provoke a state of oxidative stress, associated with pathophysiological progression in many diseases [21,22]. The intracellular changes during ischemia, including accumulation of $\mathrm{H}^{+}$and $\mathrm{Ca}^{2+}$ as well as the disruption of mitochondrial membrane potential, lead to the formation of $\operatorname{ROS}[23,24]$. Furthermore, ROS accumulation directly activates the pathways of stress response, subsequently resulting in apoptosis and impaired cellular functions[25,26]. Meanwhile, many apoptotic stimulations, including treatment with TNF- $\alpha$ [27], lipopolysaccharide (LPS)[28] and growth factor withdrawal[29] can stimulate the ROS generation by mitochondria. On the other hand, antioxidants, such as $\mathcal{N}$-acetylcysteine, thioredoxin and MnSOD can block or delay apoptosis[30]. Taken together, ROS has been considered as the trigger and the regulator of apoptosis. In the present study, our results revealed that hypoxia promoted the ROS formation and increased apoptosis in CMEGs, which is in line with the speculation for a pivotal role of oxidative stress in the onset of apoptosis in ECs[31].

FOXO regulates pro-apoptotic genes, which are necessary to induce apoptosis. Meanwhile, ROS-induced cell death was suppressed by FOXO3a mutant[32]. Moreover, FOXO family 
was strictly regulated in response to oxidative stress. On the other hand, ROS production is also modulated by the transcription activity of FoxO3a[33]. These findings indicate that FOXO is a key regulator of ROS-induced apoptosis in mammalian cells. In the present study, we observed that siRNA-target knockdown of FoxO3a suppressed hypoxia induced ROS accumulation in CMECs, indicating that hypoxia induced ROS formation is required for FoxO3a. However, these results are in conflict with those of previous studies reporting that FoxO3a regulates detoxification of ROS[33] and modulates the protective effects against oxidative stress-derived cellular damage[34]. One possible explanation for the discrepancy is that the function of FoxO proteins has been shown to be highly context-specific and may differ in different cells and stimulations. Therefore, the detailed relationship between FoxO3a and ROS stress needs further investigation.

Apoptosis is the process of programmed cell death which can be induced by the extrinsic pathway or by the intrinsic pathway. Members of the Bcl-2 family are the critical regulators of intrinsic pathway in apoptosis by controlling the balance of pro- and antiapoptotic proteins at the mitochondria[35]. Bcl-2 family is divided into pro-apoptosis subgroup (Bcl-2, Bcl-XL) and anti-apoptosis subgroup such as Bax and Bim. The ratio of pro- and antiapoptotic proteins determines cell fate at the level of mitochondria[35]. FoxO3a has been shown to mediate apoptosis by regulating the target genes including $\operatorname{Bim}[36]$. Furthermore,previous study demonstrated that FoxO3a stimulates the proapoptotic proteins Noxa and Bim, which leads to the release of cytochrome $\mathrm{c}$ in neuroblastoma cells[36]. Importantly, Bim also triggers ROS accumulation and remarkably reduces mitochondrial respiration $[37,38]$. In the present studies, we observed that hypoxia increased apoptosis in CMECs, which was abolished by inhibition of HIF- $1 \alpha$ and FoxO3a respectively. Moreover, the proapoptosis proteins Bim EL and Bax were increased, whereas, the expressions of anti-apoptotic BclxL and $\mathrm{Bcl}-2$ were decreased under hypoxia, which is in line with previous study. Furthermore, hypoxia induced disturbance of Bcl-2 family proteins was also abolished by FoxO3a silencing. These data suggest that the hypoxia-induced apoptosis is triggered by the disturbance of Bcl-2 family, which are critical downstream mediators of FoxO3.

In this study we used in vitro cell model to dissect the regulation of hypoxia injury in CMECs. Although the hypoxia model used here is useful to exclude the influence of neural and other humoral factors in vivo, this artificial model cannot fully simulate the in vivo

\section{References}

1. Braunwald E, Bristow MR (2000) Congestive heart failure: fifty years of progress. Circulation 102: IV14-23.

2. Li JM, Mullen AM, Shah AM (2001) Phenotypic properties and characteristics of superoxide production by mouse coronary microvascular endothelial cells. J Mol Cell Cardiol 33: 1119-1131.

3. Scarabelli T, Stephanou A, Rayment N, Pasini E, Comini L, et al. (2001) Apoptosis of endothelial cells precedes myocyte cell apoptosis in ischemia/ reperfusion injury. Circulation 104: 253-256.

4. Millar TM, Phan V, Tibbles LA (2007) ROS generation in endothelial hypoxia and reoxygenation stimulates MAP kinase signaling and kinase-dependent neutrophil recruitment. Free Radic Biol Med 42: 1165-1177.

5. Guzy RD, Hoyos B, Robin E, Chen H, Liu L, et al. (2005) Mitochondrial complex III is required for hypoxia-induced ROS production and cellular oxygen sensing. Cell Metab 1: 401-408.

6. Prabhakar NR, Kumar GK, Nanduri J, Semenza GL (2007) ROS signaling in systemic and cellular responses to chronic intermittent hypoxia. Antioxid Redox Signal 9: 1397-1403.

7. Kim BM, Chung HW (2007) Hypoxia/reoxygenation induces apoptosis through a ROS-mediated caspase-8/Bid/Bax pathway in human lymphocytes. Biochem Biophys Res Commun 363: 745-750.

8. Accili D, Arden KC (2004) FoxOs at the crossroads of cellular metabolism, differentiation, and transformation. Cell 117: 421-426. ischemic environment. Moreover, the detailed relationship between FoxO3a and ROS stress was not extensively elucidated in this research. Therefore, further studies are needed to thoroughly understand the mechanism of hypoxia injury in CMECs.

In conclusion, our data provide evidence for an essential role of FoxO3a in hypoxia-induced ROS formation, which is mediated by HIF-1 $\alpha$. Furthermore, FoxO3a induces Bcl-2 family disturbance which triggers the apoptosis of CMECs. This dual effect of FoxO3a seems critical in the regulation of hypoxic injury in CMECs. Taken together, these data collectively demonstrate that HIF- $1 \alpha$ induced FoxO3a activation plays a central role in hypoxia-induced ROS formation and apoptosis in CMECs.

\section{Supporting Information}

Figure S1 Real-time PGR analysis of the mRNA expressions of FoxO3a, Bim and Bcl-xL in GMECs with HIF-a silencing. Statistic analysis of the mRNA expressions of FoxO3a (A), Bim (B) and Bcl-xL (C) in CMEGs under normal or hypoxic conditions with or without HIF- $\alpha$ silencing $\left(n=5,{ }^{*} \mathrm{p}<0.05\right.$, NS: non-significant).

(TIF)

Figure S2 Real-time PGR analysis of the mRNA expressions of Bim, Bax, Bcl-2 and Bcl-xL in CMEGs with FoxO3a silencing. Statistic analysis of the mRNA expressions of $\operatorname{Bim}(\mathrm{A}), \operatorname{Bax}(\mathrm{B}), \mathrm{Bcl}-2$ (C) and Bcl-xL (D) in CMECs under normal or hypoxic conditions with or without FoxO3a silencing (n $=5,{ }^{*} \mathrm{p}<0.05$, NS: non-significant).

(TIF)

\section{Methods S1 Supplementary Methods. (DOG)}

\section{Acknowledgments}

The authors are grateful to Dr. Zhen Chen for the Western blot assays and to Dr. Lixiao Wang for his technological assistance.

\section{Author Contributions}

Conceived and designed the experiments: YY XN. Performed the experiments: SZ YilinZ QZ. Analyzed the data: LY YujieZ MX. Contributed reagents/materials/analysis tools: JC. Wrote the paper: XN.

9. van der Horst A, Burgering BM (2007) Stressing the role of FoxO proteins in lifespan and disease. Nat Rev Mol Cell Biol 8: 440-450.

10. Skurk C, Maatz H, Kim HS, Yang J, Abid MR, et al. (2004) The Akt-regulated forkhead transcription factor FOXO3a controls endothelial cell viability through modulation of the caspase-8 inhibitor FLIP. J Biol Chem 279: 1513-1525.

11. Arden KC (2007) FoxOs in tumor suppression and stem cell maintenance. Cell 128: $235-237$.

12. Samarin J, Wessel J, Cicha I, Kroening S, Warnecke C, et al. (2010) FoxO proteins mediate hypoxic induction of connective tissue growth factor in endothelial cells. J Biol Chem 285: 4328-4336.

13. Zhang Z, Li W, Sun D, Zhao L, Zhang R, et al. (2011) Toll-like receptor 4 signaling in dysfunction of cardiac microvascular endothelial cells under hypoxia/reoxygenation. Inflamm Res 60: 37-45.

14. Zhang Z, Li S, Cui M, Gao X, Sun D, et al. (2013) Rosuvastatin enhances the therapeutic efficacy of adipose-derived mesenchymal stem cells for myocardial infarction via PI3K/Akt and MEK/ERK pathways. Basic Res Cardiol 108: 333.

15. Sun D, Huang J, Zhang Z, Gao H, Li J, et al. (2012) Luteolin limits infarct size and improves cardiac function after myocardium ischemia/reperfusion injury in diabetic rats. PLoS One 7: e33491.

16. Nguyen LK, Cavadas MA, Scholz CC, Fitzpatrick SF, Bruning U, et al. (2013) A dynamic model of the hypoxia-inducible factor lalpha (HIF-1alpha) network. J Cell Sci 126: 1454-1463. 
17. Wartenberg M, Ling FC, Muschen M, Klein F, Acker H, et al. (2003) Regulation of the multidrug resistance transporter P-glycoprotein in multicellular tumor spheroids by hypoxia-inducible factor (HIF-1) and reactive oxygen species. FASEB J 17: 503-505.

18. Bonello S, Zahringer C, BelAiba RS, Djordjevic T, Hess J, et al. (2007) Reactive oxygen species activate the HIF-1alpha promoter via a functional NFkappaB site. Arterioscler Thromb Vasc Biol 27: 755-761.

19. Ronnebaum SM, Patterson C (2010) The FoxO family in cardiac function and dysfunction. Annu Rev Physiol 72: 81-94.

20. Myatt SS, Brosens JJ, Lam EW (2011) Sense and sensitivity: FOXO and ROS in cancer development and treatment. Antioxid Redox Signal 14: 675-687.

21. Ali SS, Hsiao M, Zhao HW, Dugan LL, Haddad GG, et al. (2012) Hypoxiaadaptation involves mitochondrial metabolic depression and decreased ROS leakage. PLoS One 7: e36801.

22. Gonzalez C, Sanz-Alfayate G, Agapito MT, Gomez-Nino A, Rocher A, et al. (2002) Significance of ROS in oxygen sensing in cell systems with sensitivity to physiological hypoxia. Respir Physiol Neurobiol 132: 17-41.

23. Singal PK, Bello-Klein A, Farahmand F, Sandhawalia V (2001) Oxidative stress and functional deficit in diabetic cardiomyopathy. Adv Exp Med Biol 498: 213 220.

24. Dvoriantchikova G, Grant J, Santos AR, Hernandez E, Ivanov D (2012) Neuronal $\mathrm{NAD}(\mathrm{P}) \mathrm{H}$ oxidases contribute to ROS production and mediate RGC death after ischemia. Invest Ophthalmol Vis Sci 53: 2823-2830.

25. Morey M, Corominas M, Serras F (2003) DIAP1 suppresses ROS-induced apoptosis caused by impairment of the selD/sps1 homolog in Drosophila. J Cell Sci 116: 4597-4604.

26. Sanada M, Kuroda K, Ueda M (2011) ROS production and apoptosis induction by formation of Gtslp-mediated protein aggregates. Biosci Biotechnol Biochem 75: 1546-1553.

27. Kim JJ, Lee SB, Park JK, Yoo YD (2010) TNF-alpha-induced ROS production triggering apoptosis is directly linked to Romol and $\mathrm{Bcl}-\mathrm{X}(\mathrm{L})$. Cell Death Differ 17: $1420-1434$.
28. Du SC, Ge QM, Lin N, Dong Y, Su Q (2012) ROS-mediated lipopolysaccharide-induced apoptosis in INS-1 cells by modulation of Bcl-2 and Bax. Cell Mol Biol (Noisy-le-grand) 58 Suppl: OL1654-1659.

29. Jiang L, Pan X, Chen Y, Wang K, Du Y, et al. (2011) Preferential involvement of both ROS and ceramide in fenretinide-induced apoptosis of HL60 rather than NB4 and U937 cells. Biochem Biophys Res Commun 405: 314-318.

30. Oh J, Hur MW, Lee CE (2009) SOCS1 protects protein tyrosine phosphatases by thioredoxin upregulation and attenuates Jaks to suppress ROS-mediated apoptosis. Oncogene 28: 3145-3156.

31. Kou B, Zhang J, Singer DR (2009) Effects of cyclic strain on endothelial cell apoptosis and tubulogenesis are dependent on ROS production via $\mathrm{NAD}(\mathrm{P}) \mathrm{H}$ subunit p22phox. Microvasc Res 77: 125-133.

32. Nakamura T, Sakamoto K (2008) Forkhead transcription factor FOXO subfamily is essential for reactive oxygen species-induced apoptosis. Mol Cell Endocrinol 281: 47-55.

33. Ferber EC, Peck B, Delpuech O, Bell GP, East P, et al. (2012) FOXO3a regulates reactive oxygen metabolism by inhibiting mitochondrial gene expression. Cell Death Differ 19: 968-979.

34. Kops GJ, Dansen TB, Polderman PE, Saarloos I, Wirtz KW, et al. (2002) Forkhead transcription factor FOXO3a protects quiescent cells from oxidative stress. Nature 419: 316-321.

35. Harford TJ, Shaltouki A, Weyman CM (2010) Increased expression of the proapoptotic Bcl2 family member PUMA and apoptosis by the muscle regulatory transcription factor MyoD in response to a variety of stimuli. Apoptosis 15: 7182.

36. Hagenbuchner J, Kuznetsov A, Hermann M, Hausott B, Obexer P, et al. (2012) FOXO3-induced reactive oxygen species are regulated by BCL2L11 (Bim) and SESN3. J Cell Sci 125: 1191-1203.

37. Lee BC, Park BH, Kim SY, Lee YJ (2011) Role of Bim in diallyl trisulfideinduced cytotoxicity in human cancer cells. J Cell Biochem 112: 118-127.

38. Luo H, Yang Y, Duan J, Wu P, Jiang Q, et al. (2013) PTEN-regulated AKT/ FoxO3a/Bim signaling contributes to reactive oxygen species-mediated apoptosis in selenite-treated colorectal cancer cells. Cell Death Dis 4: e481. 